\title{
Nonlinear Refraction of Peripheral-Substituted Zinc Phthalocyanines Investigated by Nanosecond and Picosecond Z Scans
}

\author{
Yunjing Li ${ }^{1}$, Timothy M. Pritchett ${ }^{2}$, Jiandong Huang ${ }^{3}$, Meirong $\mathrm{Ke}^{3}$, Wenfang Sun ${ }^{1 *}$ \\ ${ }^{1}$ Department of Chemistry and Biochemistry, North Dakota State University, Fargo, USA \\ ${ }^{2} U$. S. Army Research Laboratory, AMSRD-ARL-SE-EM, 2800 Powder Mill Road, Adelphi, Maryland, USA \\ ${ }^{3}$ College of Chemistry and Chemical Engineering, Institute of Research on Functional Materials, Fuzhou University, \\ Fuzhou, China \\ E-mail: Wenfang.Sun@ndsu.edu \\ Received April 6, 2011; revised May 9, 2011; accepted May 19, 2011
}

\begin{abstract}
The singlet and triplet excited-state refraction cross-sections of dimethyl sulfoxide (DMSO) solutions of ten zinc phthalocyanine derivatives with mono- or tetra-peripheral substituents at $532 \mathrm{~nm}$ were obtained by simultaneous fitting of closed-aperture Z scans with both nanosecond and picosecond pulse widths. Self-focusing of both nanosecond and picosecond laser pulses was observed in all complexes at 532-nm wavelength. The complexes with substituents at all of the four $\alpha$-positions exhibit relatively larger refraction cross-sections than the other complexes. The wavelength dependence of the singlet refraction cross-section of a representative complex was observed to be non-monotonic in the range of $470-550 \mathrm{~nm}$.
\end{abstract}

Keywords: Nonlinear Refraction, Zinc Phthalocyanine, Z Scan, Excited-State Refraction Cross-Section, Wavelength Dispersion

\section{Introduction}

In recent years, there has been a growing interest in materials with a large and fast nonlinear optical response for optical device applications [1-3]. Among the various organic materials investigated, metallophthalocyanines (MPcs) have attracted considerable attention because their structures can be easily modified without affecting their stability or altering their processability features [4-6]. Most recently, the photophysical properties of ten new zinc phthalocyanine derivatives with mono or tetraperipheral substituents (structures shown in Figure 1) were studied [7]. All complexes were found to exhibit reverse saturable absorption of nanosecond pulses at 532 $\mathrm{nm}$ and of picosecond pulses over a broad visible spectral range. The singlet and triplet excited-state absorption cross-sections were obtained by fitting nanosecond and picosecond open-aperture Z-scan data using a five-level model. It is found that the complexes with tetra substituents at the $\alpha$-positions exhibit larger ratios of triplet excited-state absorption to ground-state absorption crosssections $\left(\sigma_{T} / \sigma_{g}\right)$ than the other complexes; and the ratio of singlet excited-state absorption cross-section to groundstate absorption cross-section decreases from $470 \mathrm{~nm}$ to $550 \mathrm{~nm}$ [7]. This study is quite intriguing; however, no information was obtained on the nonlinear refraction of these complexes from the open-aperture Z-scan study.

In order to gain an understanding of how the peripheral substituents influence the nonlinear refractive properties of these complexes, nanosecond and picosecond closed-aperture Z-scan measurements were carried out in this study. Using the previously measured values of excited-state absorption cross-sections, values of the singlet and the triplet excited-state refraction cross-sections were determined from the closed-aperture $Z$-scan data; the results are reported in this paper. In addition, the wavelength dependence of the singlet excited-state refraction cross-section over a range extending from $470 \mathrm{~nm}$ to $550 \mathrm{~nm}$ was studied using picosecond closed-aperture Z scans.

\section{Experimental}

The Z-scan experimental setup was described previously [7]. A Quantel Brilliant Nd: YAG laser operating at its 

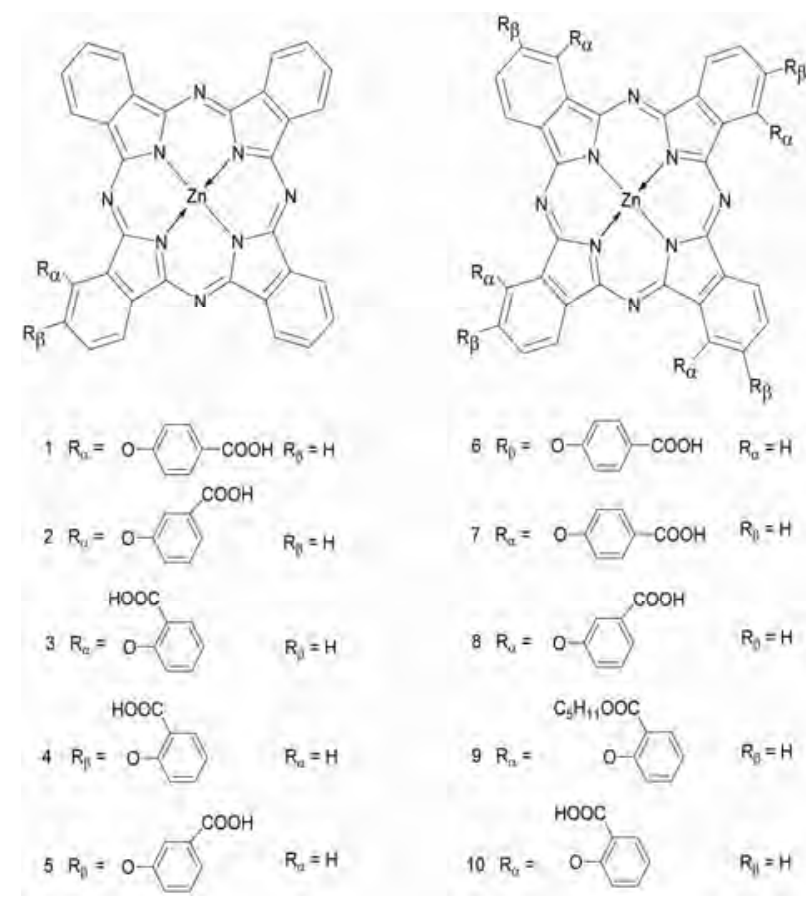

Figure 1. Structures of ZnPc derivatives 1 - 10.

second-harmonic output $(4.1 \mathrm{~ns}, 10 \mathrm{~Hz})$ and a picosecond EKSPLA PG 401 optical parametric generator (OPG) pumped by the third-harmonic output of an EKSPLA PL 2143A Nd:YAG laser (21 ps, $10 \mathrm{~Hz}$ ) were used for the closed-aperture Z-scan experiments. The $\mathrm{HWe}^{-2} \mathrm{M}$ beam waist was measured to be $32 \mu \mathrm{m}$ for the nanosecond $\mathrm{Z}$ scans and $40 \mu \mathrm{m}$ at $532 \mathrm{~nm}$ for the picosecond Z scans by knife edge. The sample solutions were placed in a 2-mm quartz cuvette for the ps measurements and in a 1-mm cuvette for the ns measurements, which were less than the Rayleigh ranges to fulfill the thin sample approximation condition. A small aperture $(S=0.22$ for the ns measurement and 0.35 for the ps measurement at 532 $\mathrm{nm}$, and $S=0.34-0.42$ for the ps measurements from $470 \mathrm{~nm}$ to $570 \mathrm{~nm}$ ) was placed before the detector at the far field for the closed aperture experiments.

In order to abstract the nonlinear refraction cross- section, a five-level model described previously [7] was used to fit the open-aperture and the closed-aperture curves. First, the values of the excited-state absorption cross-sections for the singlet and triplet excited states $\left(\sigma_{\mathrm{S}}\right.$ and $\sigma_{\mathrm{T}}$, respectively) were obtained by simultaneously fitting nanosecond and picosecond open-aperture $\mathrm{Z}$ scans at $532 \mathrm{~nm}$, in which the effects of excited-state refraction are absent, using the five-band model that employed independently measured values of the excited-state lifetimes and of the triplet yield. The values of $\sigma_{S}$ and $\sigma_{T}$ obtained from the open-aperture Z scans, together with the measured values of the excited-state lifetimes and of the triplet yield, were then reinserted in a generalized five-band model that includes the effects of both excited-state refraction and excited-state absorption.

The effects of excited-state refraction are described mathematically by the nonlinear phase $\phi$ computed at each point on the exit face of the sample from the following equation:

$$
\frac{\partial \phi}{\partial z}=\sigma_{S}^{(r)} n_{S}+\sigma_{T}^{(r)} n_{T}
$$

Where $\sigma_{S}^{(r)}$ and $\sigma_{T}^{(r)}$ are the refraction cross-sections of the singlet excited state and the lowest-lying triplet excited state, respectively. The number densities of molecules in these states, denoted by $n_{S}$, and $n_{T}$ respectively, are functions of both the position $(r, z)$ in the sample and the time $t$; they are computed as described in [7]. For each ZnPc derivative, a single pair of refractive cross-section values $\left(\sigma_{S}^{(r)}, \sigma_{T}^{(r)}\right)$ was chosen to simultaneously fit both the picosecond and the nanosecond closed-aperture Z scans. In fitting closed-aperture Z scans at wavelengths other than $532 \mathrm{~nm}$, the value of $\sigma_{T}^{(r)}$ was assumed to be the same as that obtained from the simultaneous fitting of the corresponding 532-nm Z scans.

\section{Results and Discussion}

The electronic absorption spectra of complexes 1 - 10 in dimethyl sulfoxide (DMSO) have been reported previously [7]. Figure 2 shows the absorption spectrum of complex 5, which is fairly typical of the spectra of all ten complexes. As noted in [7], all complexes possess a fairly broad "transparency window" between the B-bands and Q-bands (425 nm and $575 \mathrm{~nm}$, respectively), and all complexes exhibit strong reverse saturable absorption for both nanosecond and picosecond laser pulses at $532 \mathrm{~nm}$.

Figure 3 shows the closed-aperture Z-scan curves for complex 6, which are representative of all ten ZnPc derivatives. The valley-peak feature is indicative of a positive refraction nonlinearity (self-focusing). The profile is strongly asymmetric about a normalized transmittance of unity, above which the peak is almost completely

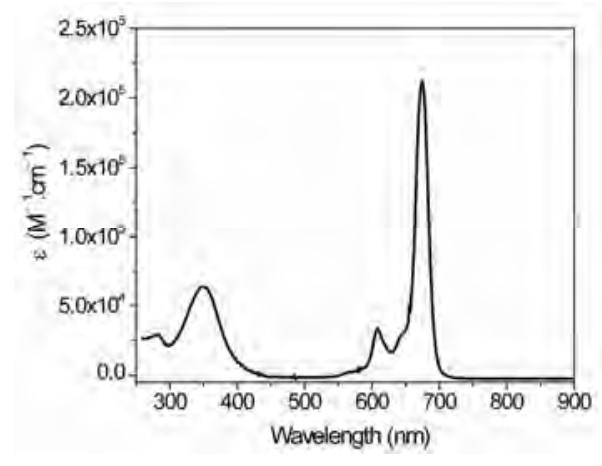

Figure 2. UV-vis absorption spectrum of complex 5 in DMSO. 


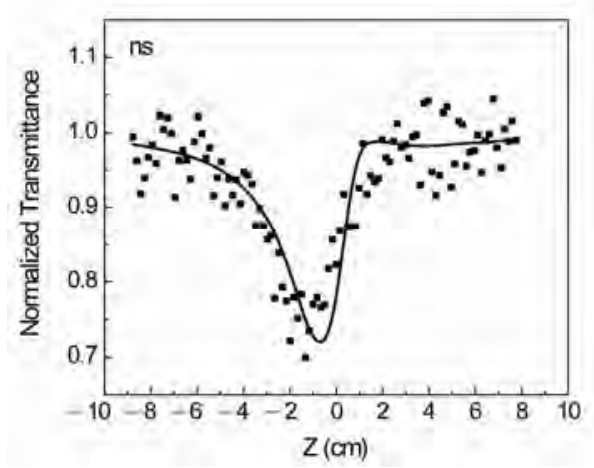

(a)

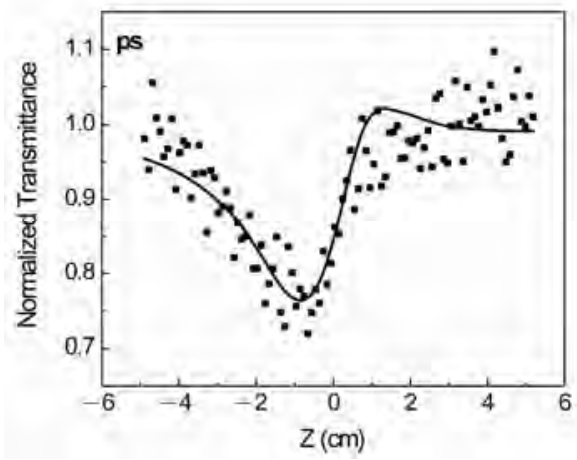

(b)

Figure 3. Normalized nanosecond and picosecond closedaperture Z-scan data (squares) and fitting curves (solid lines) for complex 6 in DMSO at $532 \mathrm{~nm}$.

suppressed. The asymmetry arises from the presence of an absorptive nonlinearity (reverse saturable absorption). Our previously published open-aperture Z-scan results demonstrate that all of the ZnPc derivatives display significant reverse saturable absorption [7].

For compounds possess both nonlinear absorption and nonlinear refraction, a simple division of the closed-aperture Z-scan curve by that obtained with the aperture removed (open-aperture) does not necessarily provide a good approximation of the curve that would be obtained with a closed-aperture $\mathrm{Z}$ scan of a material having no nonlinear absorption [8,9]. For this reason, the two-step procedure described in the experimental section was employed to obtain the fitting curves shown by solid lines in Figure 3. The solid curves in Figure 3 represent the best fit of the experimental Z-scan data for complex 6 and correspond to the values of $\sigma_{\mathrm{S}}^{(r)}=(5.0 \pm 1.0) \times 10^{-17}$ $\mathrm{cm}^{2}$ and $\sigma_{T}^{(r)}=(1.0 \pm 0.5) \times 10^{-17} \mathrm{~cm}^{2}$.

Table 1 lists the absorption and refraction cross-sections of the singlet excited state and the triplet excited state for the ten ZnPc derivatives in DMSO. The magnitudes of the excited-state refraction cross-sections are all on the order of $10^{-17}-10^{-16} \mathrm{~cm}^{2}$. The three $\alpha$-tetrasubstituted complexes 8, 9 and 10 all possess much larger triplet refractive cross-sections than either the mono substituted complexes (1-5) or the $\beta$-tetrasubstituted complex (6), which is similar to the trend displayed by the triplet excited-state absorption cross-sections. This phenomenon could be explained by the geometry of the $\alpha$ - and $\beta$-tetrasubstituted complexes. As shown in Figure 4, the geometry-optimized structures for complexes 6 and 7 (obtained via B3LYP/3-21g level density functional theory (DFT) calculation in vacuum using Gaussian 09) are drastically different. The steric hindrance imposed by the substituents at the $\alpha$-positions makes the substituents in complex 7 adopt a nearly perpendicular geometry to the phthalocyanine ring. This makes complex 7 bulkier than complex 6 , which has the substituents at the $\beta$-positions. The bulkiness of complex 7 would reduce intermolecular interactions, which prevents excitation quenching and stabilizes the excited state. Consequently, the nonlinearities of the complex are higher than those with larger extent of intermolecular aggregation [10]. The same effect holds for complexes 8, 9 and 10. In contrast, the singlet excited-state refraction cross-sections do not vary significantly among these ten complexes.

The wavelength dependence of the excited-state refraction cross-section of complex 5 was studied at multiple visible wavelengths; it was found to exhibit self-focusing nonlinear refraction in the range of $470 \mathrm{~nm}$ to $570 \mathrm{~nm}$. Assuming $\sigma_{T}^{(r)}$ values at other wavelengths equal to the value obtained at $532 \mathrm{~nm}\left(6.0 \times 10^{-17} \mathrm{~cm}^{2}\right)$, the following values of $\sigma_{s}^{(r)}$ at wavelengths from $470 \mathrm{~nm}$ to $550 \mathrm{~nm}$ were obtained by fitting the relevant ps $\mathrm{Z}$ scans of complex 5: $4.0 \times 10^{-17} \mathrm{~cm}^{2}(470 \mathrm{~nm}), 3.0 \times 10^{-17} \mathrm{~cm}^{2}(500 \mathrm{~nm})$ and $5.0 \times 10^{-17} \mathrm{~cm}^{2}(550 \mathrm{~nm})$. The parameters used for

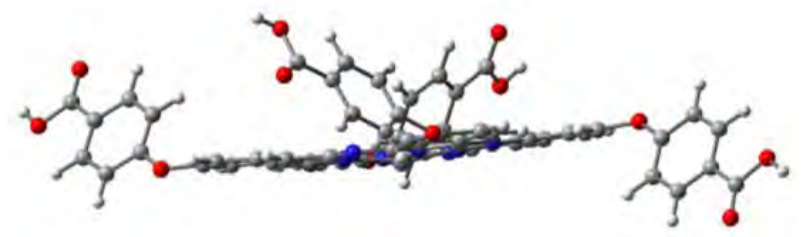

(a)

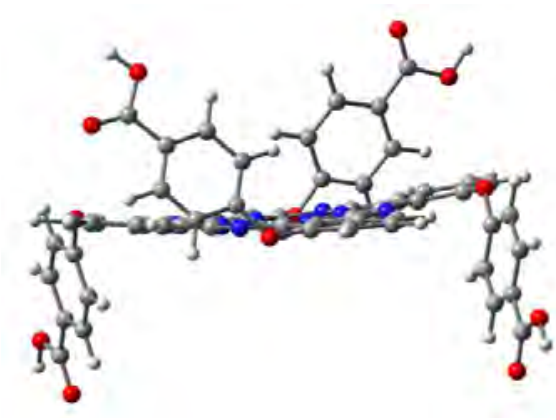

(b)

Figure 4. Geometry-optimized structures for complexes 6 (upper) and 7 (lower) via DFT calculation. 
Table 1. Best-fit excited-state parameters of ZnPc derivatives 1 - 10 in DMSO.

\begin{tabular}{|c|c|c|c|c|c|c|}
\hline & $\begin{array}{c}\sigma_{\mathrm{g}}^{*} \\
/ 10^{-18} \mathrm{~cm}^{2}\end{array}$ & $\begin{array}{c}\sigma_{S}^{*} \\
/ 10^{-18} \mathrm{~cm}^{2}\end{array}$ & $\begin{array}{c}\sigma_{T}{ }^{*} \\
/ 10^{-18} \mathrm{~cm}^{2} \\
\end{array}$ & $\begin{array}{c}\sigma_{S}^{(r) \#} \\
/ 10^{-18} \mathrm{~cm}^{2}\end{array}$ & $\begin{array}{c}\sigma_{T}^{(r) \#} \\
/ 10^{-18} \mathrm{~cm}^{2}\end{array}$ & $\begin{array}{l}\tau_{S}^{*} \\
/ \mathrm{ns} \\
\end{array}$ \\
\hline 1 & 3.1 & $40 \pm 5$ & $180 \pm 20$ & $40 \pm 10$ & $150 \pm 20$ & 3.01 \\
\hline 2 & 2.4 & $40 \pm 5$ & $105 \pm 20$ & $40 \pm 5$ & $80 \pm 10$ & 3.11 \\
\hline 3 & 2.5 & $45 \pm 5$ & $70 \pm 15$ & $35 \pm 5$ & $100 \pm 20$ & 3.10 \\
\hline 4 & 3.7 & $45 \pm 8$ & $110 \pm 20$ & $50 \pm 10$ & $60 \pm 10$ & 3.17 \\
\hline 5 & 3.1 & $45 \pm 5$ & $60 \pm 15$ & $60 \pm 10$ & $60 \pm 10$ & 3.27 \\
\hline 6 & 4.0 & $40 \pm 6$ & $45 \pm 10$ & $50 \pm 10$ & $10 \pm 5$ & 3.00 \\
\hline 7 & 1.6 & $30 \pm 5$ & $170 \pm 20$ & $50 \pm 10$ & $60 \pm 5$ & 2.70 \\
\hline 8 & 2.3 & $18 \pm 4$ & $180 \pm 25$ & $40 \pm 10$ & $160 \pm 20$ & 2.70 \\
\hline 9 & 1.3 & $18 \pm 2$ & $400 \pm 40$ & $25 \pm 5$ & $250 \pm 50$ & 2.59 \\
\hline 10 & 1.8 & $30 \pm 5$ & $280 \pm 30$ & $40 \pm 10$ & $300 \pm 50$ & 2.59 \\
\hline
\end{tabular}

*From Reference 7; ${ }^{*}$ This work.

Table 2. Best-fit excited-state parameters of ZnPc derivative 5 in DMSO at various wavelengths.

\begin{tabular}{|c|c|c|c|c|c|}
\hline$\lambda / \mathrm{nm}$ & $\begin{array}{c}\sigma_{g}^{*} \\
/ 10^{-19} \mathrm{~cm}^{2}\end{array}$ & $\begin{array}{c}\sigma_{S}^{*} \\
/ 10^{-18} \mathrm{~cm}^{2}\end{array}$ & $\begin{array}{c}\sigma_{T}{ }^{*} \\
/ 10^{-18} \mathrm{~cm}^{2} \\
\end{array}$ & $\begin{array}{c}\sigma_{S}^{(r) \#} \\
/ 10^{-18} \mathrm{~cm}^{2}\end{array}$ & $\begin{array}{c}\sigma_{T}^{(r) \#} \\
/ 10^{-18} \mathrm{~cm}^{2} \\
\end{array}$ \\
\hline 470 & 4.6 & $40 \pm 8$ & $60 \pm 15$ & $40 \pm 20$ & $60 \pm 10$ \\
\hline 500 & 5.5 & $32 \pm 6$ & $60 \pm 15$ & $30 \pm 10$ & $60 \pm 10$ \\
\hline 532 & 31.0 & $45 \pm 5$ & $60 \pm 15$ & $60 \pm 10$ & $60 \pm 10$ \\
\hline 550 & 76.0 & $40 \pm 10$ & $60 \pm 15$ & $50 \pm 10$ & $60 \pm 10$ \\
\hline
\end{tabular}

"From [7]; " This work.

the fitting are summarized in Table 2. No significant dispersion of the nonlinear refraction cross-section was observed for 5 in the spectral region studied.

Linear absorption by the sample creates a thermal lens whose rise time is given by $w_{0} / c_{s}$, where $w_{0}$ is the radius of the focal spot and $c_{s}$ is the speed of sound in the solvent used (in the present case, $1493 \mathrm{~m} / \mathrm{s}$ ). However, since these rise times (26.8 ns for the picosecond $\mathrm{Z}$ scans and $21.4 \mathrm{~ns}$ for the nanosecond scans) are much longer than the corresponding pulse widths (21 ps and $4.1 \mathrm{~ns}$, respectively), thermal lensing can be ignored in fitting the closed-aperture $\mathrm{Z}$ scans.

\section{Conclusions}

The singlet and triplet excited-state refraction cross- sections at $532 \mathrm{~nm}$ of ten novel zinc phthalocyanine derivatives with mono- or tetra-peripheral substituent(s) in DMSO solution were obtained by using a five-level dynamic model to fit nanosecond and picosecond closedaperture Z-scan data. These results, in combination with those for the singlet and triplet excited-state absorption cross-sections previously obtained from open-aperture Z scans, provide a complete picture of the excited-state optical nonlinearities of the ten zinc phthalocyanine derivatives. It has been demonstrated that both the number and the position of the peripheral substituents dramatically affect the triplet excited-state absorption and refraction cross-sections. In addition, the wavelength-dependence of the singlet excited-state refraction cross-section of a representative complex was investigated over the range from 470 to $550 \mathrm{~nm}$. No monotonic dependence of refraction cross-section was observed.

\section{Acknowledgements}

W. Sun acknowledges the financial support from the NSF NIRT program (DMI 0506531) and is grateful to Dr. Joy Haley at the Air Force Research Laboratory for her help in the singlet excited-state lifetime measurement. Sun is also grateful to Professor Hongshan $\mathrm{He}$ at the South Dakota State University for his kind help in DFT calculations to obtain the geometry-optimized structures for complexes 6 and 7. J. Huang acknowledges the financial support from the National Natural Science Foundation of China (Grant No. 20201005).

\section{References}

[1] P. N. Prasad and D. J. Williams, "Introduction to Nonlinear Optics Effects in Molecules and Polymers," Wiley, New York, 1991.

[2] P. Shao, Y. Li and W. Sun, “Cyclometalated Platinum (II) Complex with Strong and Broadband Nonlinear Optical Response,” Journal of Physical Chemistry A, Vol. 112, No. 6, 2008, pp. 1172-1179. doi:10.1021/jp710616z

[3] S. M. O’Flaherty, J. J. Doyle and W. J. Blau, "Numerical Approach for Optically Limited Pulse Transmission in 
Polymer-Phthalocyanine Composite Systems,” The Journal of Physical Chemistry B, Vol. 108, No. 45, 2004, 17313-17319. doi:10.1021/jp047005t

[4] H. S. Nalwa, and J. S. Shirk, In: C. C. Leznoff and A. B. P. Lever, Eds., Phthalocyanines: Properties and Applications, VCH Publishers, Inc., New York, Vol. 4, 1996, p. 83.

[5] X. À. Micó, S. I. Vagin, L. R. Subramnian, T. Ziegler and M. Hanack, "New Unsymmetrical Zinc-Phthalocyanine Conjugated with One Azo-Dye Moiety: Synthesis Via Opening the Fused Triazole Ring and Spectral Properties,” European Journal of Organic Chemistry, Vol. 2005, No. 20, 2005, 4328-4337. doi:10.1002/ejoc.200500237

[6] N. B. Mckeown, "Phthalocyanine Materials: Synthesis, Structure and Function," Cambridge University Press, Cambridge, 1998.

[7] Y. Li, T. M. Pritchett, J. Huang, M. Ke, P. Shao and W. Sun, "Photophysics and Nonlinear Absorption of Periph- eral Substituted Zinc Phthalocyanines,” Journal of Physical Chemistry A, Vol. 112, No. 31, 2008, pp. 7200-7207. doi:10.1021/jp7108835

[8] M. Sheik-Bahae, A. A. Said, T. H. Wei, D. J. Hagan, M. J. Soileau and E. W. van Stryland, "Sensitive Measurement of Optical Nonlinearities Using a Single Beam,” IEEE Journal of Quantum Electronics, Vol. 26, No. 4, 1990, pp. 760-769. doi:10.1109/3.53394

[9] E. W. van Stryland and M. Sheik-Bahae, "Z-Scan”, In: M. Kuzyk and C. Dirk, Eds., Characterization Techniques and Tabulations for Organic Nonlinear Optical Materials, Marcel Decker, Basel, 1998, p. 663.

[10] J. S. Shirk, R. G. S. Pong, S. R. Flom, H. Heckmann and M. Hanack, "Effect of Axial Substitution on the Optical Limiting Properties of Indium Phthalocyanines," Journal of Physical Chemistry A, Vol. 104, No. 7, 2000, pp. 1438-1449. doi:10.1021/jp993254j 